\title{
Correction to: New perspectives in nanotherapeutics for chronic respiratory diseases
}

\author{
Adriana Lopes da Silva ${ }^{1,2} \cdot$ Fernanda Ferreira $\mathrm{Cruz}^{1} \cdot$ Patricia Rieken Macedo Rocco $^{1}$. \\ Marcelo Marcos Morales ${ }^{2}$
}

Published online: 6 October 2017

(C) International Union for Pure and Applied Biophysics (IUPAB) and Springer-Verlag GmbH Germany 2017

\section{Correction to: Biophys Rev}

https://doi.org/10.1007/s12551-017-0319-x

The original version of this article unfortunately contains an error. The third author's name "Patricia Rieken Macedo Rocco" was incorrectly spelled with "Roccco". The correct author name is now presented in the authorgroup.

The original article has been corrected.

The online version of the original article can be found at https://doi.org/ 10.1007/s12551-017-0319-x

\section{Marcelo Marcos Morales}

mmorales@biof.ufrj.br

1 Laboratory of Pulmonary Investigation, Carlos Chagas Filho Institute of Biophysics, Federal University of Rio de Janeiro, Rio de Janeiro, RJ, Brazil

2 Laboratory of Cellular and Molecular Physiology, Carlos Chagas Filho Institute of Biophysics, Federal University of Rio de Janeiro, Centro de Ciências da Saúde, Avenida Carlos Chagas Filho, s/n, Bloco G, Ilha do Fundão, Rio de Janeiro, RJ 21941-902, Brazil 